\title{
Reviewers in this Issue
}

Dr. M. A. Salam Khan

Professor of Anaesthesiology, SICU

BIRDEM Academy and Hospital, Dhaka, Bangladesh

Dr. Shafiqul Alam

Professor of Ophthalmology

Delta Medical College, Dhaka, Bangladesh

Dr. S. M. Fazlul Karim

Professor of Biochemistry

Delta Medical College, Dhaka, Bangladesh

Dr. Md. Nur Hossain

Professor of Forensic Medicine

Delta Medical College, Dhaka, Bangladesh

Dr. Md. Rezwanur Rahman

Professor of Biochemistry

Delta Medical College, Dhaka, Bangladesh

Dr. Rehnuma Jahan

Consultant of Obs and Gynae

Square Hospital, Dhaka, Bangladesh

Dr. Ahmedul Kabir

Associate Professor of Medicine

Dhaka Medical College, Dhaka, Bangladesh

Dr. Md. Robed Amin

Associate Professor of Medicine

Dhaka Medical College, Dhaka, Bangladesh 